\title{
miR-367 promotes epithelial-to-mesenchymal transition and invasion of pancreatic ductal adenocarcinoma cells by targeting the Smad7-TGF- $\beta$ signalling pathway
}

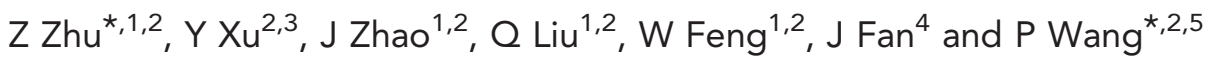

${ }^{1}$ Department of Radiation Oncology, Fudan University Shanghai Cancer Center, 270 Dong An Road, Shanghai 200032, China; ${ }^{2}$ Department of Oncology, Shanghai Medical College, Fudan University, 130 Dong An Road, Shanghai 200032, China; ${ }^{3}$ Department of Pancreatic and Hepatobiliary Surgery, Fudan University Shanghai Cancer Center, 270 Dong An Road, Shanghai 200032, China; ${ }^{4}$ Department of Pathology, Huashan Hospital, Fudan University, 12 Central Wulumuqi Road, Shanghai 200040, China and ${ }^{5}$ Department of Integrative Oncology, Fudan University Shanghai Cancer Center, 270 Dong An Road, Shanghai 200032, China

Background: Aberrant Smad7 expression contributes to the invasion and metastasis of pancreatic cancer cells. However, the potential mechanism underlying aberrant Smad7 expression in human pancreatic ductal adenocarcinoma (PDAC) remains largely unknown.

Methods: Bioinformatic prediction programmes and luciferase reporter assay were used to identify microRNAs regulating Smad7. The association between miR-367 expression and the overall survival of PDAC patients was evaluated by Kaplan-Meier analysis. The effects of miR-367 and Smad7 on the invasion and metastasis of pancreatic cancer cells were investigated both in vitro and in vivo.

Results: We found that miR-367 downregulated Smad7 expression by directly targeting its $3^{\prime}$-UTR in human pancreatic cancer cells. High level of miR-367 expression correlated with poor prognosis of PDAC patients. Functional studies showed that miR-367 promoted pancreatic cancer invasion in vitro and metastasis in vivo through downregulating Smad7. In addition, we showed that miR-367 promoted epithelial-to-mesenchymal transition by increasing transforming growth factor- $\beta$ (TGF- $\beta$ )-induced transcriptional activity.

Conclusions: The present study identified and characterised a signalling pathway, the miR-367/Smad7-TGF- $\beta$ pathway, which is involved in the invasion and metastasis of pancreatic cancer cells. Our results suggest that miR-367 may be a promising therapeutic target for the treatment of human pancreatic cancer.

Pancreatic cancer remains a major health problem with a 5-year survival rate of $<5 \%$ (Siegel et al, 2014). Although surgical resection is effective for the treatment of pancreatic cancer at an early stage, more than $80 \%$ of pancreatic cancer patients are diagnosed at a late stage when curative surgical treatment is not possible. The poor prognosis of pancreatic cancer mainly results from early invasion of cancer cells to adjacent tissues and metastasis (Siegel et al, 2014). Therefore, novel therapeutic approaches preventing cancer cell invasion and metastasis is highlighted for pancreatic cancer treatments.

It has been reported that Smad7 antagonised transforming growth factor- $\beta$ (TGF- $\beta$ ) signalling through several mechanisms, 
including binding to the $\mathrm{T} \beta \mathrm{RI}$ receptor complex and competitively inhibiting phosphorylation of R-Smads, recruiting phosphatases that dephosphorylate and inactivate the receptor complex, and activating the catalytic activity of Smurf ubiquitin ligases, leading to ubiquitylation of T $\beta$ RI (Hata et al, 1998; Ebisawa et al, 2001; Bai and Cao, 2002; Shi et al, 2004). Studies have shown that Smad7 inhibited invasion and metastasis of cancer cells triggered by TGF$\beta 1$ (Hata et al, 1998; Ebisawa et al, 2001; Bai and Cao, 2002; Shi et al, 2004). For example, adenoviral delivery of Smad7 to metastatic breast carcinoma cells led to a significant inhibition of cancer metastasis in xenografted mice (Azuma et al, 2005). Moreover, Smad7 overexpression delayed the establishment and growth of melanoma in bone tissues in a mouse model (Javelaud et al, 2005). In our previous study, we found that reduced expression of Smad7 correlated with lymph node metastasis, liver metastasis after surgery, and a poor survival rate of pancreatic cancer patients (Wang et al, 2009b), suggesting that aberrant Smad7 expression contributes to invasion and metastasis of pancreatic cancer. However, the potential mechanism underlying aberrant Smad7 expression in human pancreatic cancer remained unknown.

MicroRNAs (miRNAs) are a class of small ( $\sim 22-n t)$, noncoding, RNA molecules that function in the post-transcriptional regulation of gene expression. These molecules downregulate protein expression by binding to partially complementary sequences in mRNAs and degrading and/or inhibiting mRNA translation (Kim et al, 2009). Increasing evidence suggests that aberrant miRNA expression is involved in invasion and metastasis of pancreatic cancer (Papaconstantinou et al, 2012; Wang et al, $2014 \mathrm{~b}$ ). Given the critical role of Smad7 in invasion and metastasis of pancreatic cancer, we hypothesise that Smad7 is regulated by miRNA(s) at the post-transcriptional level. In the present study, we identified a miRNA, miR-367, that regulated Smad7 expression in human pancreatic cancer cells. We also found that miR-367 promoted invasion and metastasis of pancreatic cancer cells through direct regulation of Smad7 and the downstream TGF- $\beta$ signalling.

\section{MATERIALS AND METHODS}

Patients and clinical specimens. Human pancreatic cancer samples were collected from 65 patients after surgical resection at Huashan Hospital, Fudan University, Shanghai, China, from January 2003 to December 2005. Written informed consent was obtained from the patients, in accordance with the institutional guidelines, before sample collection, and the study was approved by the Committees for the Ethical Review of Research at the Fudan University Shanghai Cancer Center. All of the patients underwent macroscopically curative resection. Resected primary tumours and lymph nodes were histologically examined by haematoxylin and eosin staining using the tumour-node-metastasis classification system. Histologically, all of the tumours were invasive ductal adenocarcinomas. None of the patients had received neoadjuvant radio/chemotherapy. Fresh-frozen and/or formalin-fixed paraffinembedded samples were used for miR-367 and Smad7 expression analysis. Laser Microdissection approach to separate the epithelial tumour cell from surrounding microenvironment was performed on 65 samples from pancreatic ductal adenocarcinoma (PDAC) patients. The patients' characteristics are listed in Supplementary Table 1.

Cell lines and mice. The human pancreatic cancer cell lines PANC-1 and BxPC3 were obtained from the American Type Culture Collection (Manassas, VA, USA) and grown in complete growth medium as recommended by the manufacturer. The cancer cells were maintained in a humidified $5 \% \mathrm{CO}_{2}$ atmosphere at $37^{\circ} \mathrm{C}$. All cell lines were regularly authenticated by checking their morphology and confirming the absence of mycoplasma contamination (MycoAlert, Lonza, Rockland, ME, USA).

Male NOD/SCID mice (5-week-old) were obtained from the Shanghai Institute of Materia Medica, Chinese Academy of Sciences (Shanghai, China), and housed in laminar flow cabinets under specific pathogen-free conditions with food and water ad libitum. All mouse experiments were conducted in accordance with the guidelines of the NIH for the Care and Use of Laboratory Animals. The study protocol was approved by the Committee on the Use of Live Animals in Teaching and Research, Fudan University, Shanghai.

RNA isolation. Total RNA was isolated from freshly frozen tissue samples and cell lines using TRIzol Reagent (Invitrogen, San Diego, CA, USA) and small RNA enrichment was conducted using a mirVana miRNA isolation kit (Ambion Inc., Austin, TX, USA), according to the manufacturers' instructions as described previously (Lekanne Deprez et al, 2002).

qRT-PCR assays. Real-time PCR was performed to evaluate the level of miR-367 using specific TaqMan primers through the determination and comparison of mean $C_{\mathrm{T}}$ values. Briefly, $2 \mu \mathrm{l}$ aliquot of enriched small RNAs from cells or cancer tissues were reverse transcribed to cDNA using the TaqMan MicroRNA Reverse Transcription Kit (Applied Biosystems, San Diego, CA, USA) according to the manufacturer's protocol. Then $2 \mu \mathrm{l}$ cDNA was used as a template for the quantitative PCR amplification. Quantitative PCR reaction was performed using $10 \mu \mathrm{l}$ TaqMan Universal Master Mix $(2 \times$; Applied Biosystems), $1 \mu$ l gene-specific primers/probe, and nuclease-free $\mathrm{H}_{2} \mathrm{O}$ in a final volume of $20 \mu \mathrm{l}$. No-template controls were included in both steps to ensure targetspecific amplification. Quantitative PCR was run on a $7500 \mathrm{HT}$ quantitative PCR machine (Applied Biosystems). The $C_{\mathrm{T}}$ values of different samples were compared using the $\Delta \Delta C_{\mathrm{T}}$ method as previously described (Giovannetti et al, 2010). Semi-quantitative real-time RT-PCR using SYBR Green I was performed to compare the relative expression levels of specific mRNAs as previously described (Lekanne Deprez et al, 2002).

Oligonucleotide transfection. For transfection experiments, miRNA duplexes corresponding to the indicated miRNAs were designed as previously described (Lim et al, 2005). The negative control (NC) RNA duplexes for the miRNA mimics and the smallinterfering RNAs (siRNAs) were not homologous to any known human gene sequences. The siRNAs were designed to target the human Smad7 transcript (GenBank Access No: NM_001190821). All RNA oligoribonucleotides were purchased from Genepharma (Shanghai, China). The sequences were as follows: miR-367 mimics, $5^{\prime}$-UAGCUUAUCAGACUGAUGUUGA- ${ }^{\prime}$ and $5^{\prime}$-AACA UCAGUCUGAUAAGCUAUU-3'; NC, 5'-UUCUCCGAACGUG UCACGUTT- $3^{\prime}$ and $5^{\prime}$-ACGUGACACGUUCGGAGAATT- ${ }^{\prime}$; Smad7 siRNA, 5'-GGTGGCCTTGTGATCAATGAAACT-3' (sense); Anti-miR-367 (an inhibitor of miR-367), 5'-UCAACAUC AGUCUGAUAAGCUA-3'; Anti-miR-C (used as a NC for anti-miR-367 in the antagonism experiment), $5^{\prime}$-GUGGAUAUUG UUGCCAUCA-3'. The oligonucleotide transfection was performed using the Lipofectamine 2000 reagent (Invitrogen). Fifty nanomoles per litre of RNA duplex were used for each transfection.

Lentivirus transduction. Lentivirus with miR-367 overexpressing vector was purchased from GenePharma (Shanghai, China; LV3pGLVH1/GFP + Puro vector, D06003). Infection of the lentivirus into PANC-1 and BxPC3 cells was conducted under the instruction of GenePharma Recombinant Lentivirus Operation Manual (GenePharma). At $72 \mathrm{~h}$ after lentivirus infection, GFP-positive cells were sorted by flow cytometry (BD Biosciences, Franklin Lakes, NJ, USA) to select for cells with stable expression of miR-367. 
Vector constructs. To generate the Smad7 expression vector, the open reading frame of the human Smad7 gene was amplified and cloned into the pcDNA3.0 vector. Luciferase constructs were generated by ligating oligonucleotides containing the wild type or mutant putative target site of the Smad7 $3^{\prime}$-UTR into the PsiCHECK2 vector (Promega) downstream of the luciferase gene.

Luciferase assay. HEK293 and PANC-1 cells were co-transfected with $80 \mathrm{ng}$ luciferase reporter plasmid, $40 \mathrm{ng}$ pRL-TK-Renillaluciferase plasmid (Promega, Madison, WI, USA), and the indicated RNAs (final concentration: $20 \mathrm{nM}$ ). Twenty-four hours after transfection, firefly and Renilla luciferase activities were measured using a Dual-Luciferase assay kit (Promega). Each transfection was performed in triplicate and repeated twice.

Migration and invasion assay. Cell migration and invasion were evaluated using Transwell cell migration plates (Corning Inc., Corning, NY, USA) and Matrigel invasion chambers (Matrigelcoated membrane, BD Biosciences). Cells $\left(1.0 \times 10^{4}\right)$ were seeded in serum-free medium into the upper chamber and allowed to invade towards $10 \%$ FCS in the lower chamber as a chemoattractant. After $8 \mathrm{~h}$ (for migration assays without Matrigel coating) or $48 \mathrm{~h}$ (for invasion assays with matrigel coating), the cells that invaded through the membrane and adhered to the underside of the membrane were counted as previously described (Wang et al, 2009a).

Experimental metastasis assay. An in vivo metastasis model was established as previously described (Wang et al, 2014b). Briefly, NOD/SCID mice were anaesthetised with chloral hydrate; the spleen was exposed; and pancreatic cancer cells $\left(2 \times 10^{6} / 0.2 \mathrm{ml}\right.$ PBS) were injected into the spleen with a syringe. The spleen was then returned to abdomen, and the wound was closed in one layer with wound clips. Eight weeks after injection, the mice were grossly examined at necropsy for metastases in the livers. We then counted the number of metastatic colonies in one histological section of the midportion of each liver specimen. Tumour metastasis in the livers was evaluated based on the ratio of metastatic area to the total area on histological sections examined (Wang et al, 2014b). The ratio of the metastatic area to the total area was calculated with Adobe Photoshop version 7.0 (Adobe Systems) and Image J version 1.29 (National Institutes of Health) software. The results are expressed as percentages.

Western blot analysis. Western blot analysis was performed as described in our previous report (Wang et al, 2009a). Briefly, proteins were extracted from the cultured cells and quantified using the bicinchoninic acid assay kit (Pierce, Rockford, IL, USA) with BSA as a standard. Equal amounts of protein from different cells were separated by $10 \%$ SDS-PAGE and transferred to a nitrocellulose membrane (Bio-Rad, Hercules, CA, USA). The membranes were blocked with 5\% non-fat milk and incubated with primary antibodies. The target proteins were detected using an enhanced chemiluminescence kit (Amersham Pharmacia Biotech, Uppsala, Sweden). The following primary antibodies were used: anti-Smad7, anti-Smad2, anti-Smad3, anti-Smad4, anti-E-cadherin, anti-Vimentin, anti-a-SMA, anti-Desmin (Epitomics), antiphosphorylated Smad2/3 (Santa Cruz Biotechnology, Inc., Santa Cruz, CA, USA), and anti-GAPDH (ProteinTech Group, Inc., Chicago, IL, USA).

Immunofluorescence. Cells were fixed with $4 \%$ paraformaldehyde. The cells were then incubated with anti-Vimentin $(1: 200)$ and anti-E-cadherin $(1: 100)$ at $4{ }^{\circ} \mathrm{C}$ overnight, and then incubated with fluorescence-conjugated secondary antibody for $1 \mathrm{~h}$. Finally, the cells were washed and mounted with mounting medium containing DAPI (Vector Laboratories, Burlingame, CA, USA). A NC in which the primary antibody was omitted was used to test for antibody specificity. Images were captured using a nonconfocal Leica fluorescence microscope.
Statistical analysis. All results were expressed as the mean \pm s.d. The ANOVA and Student's $t$-tests were used to analyse significant differences between samples. The correlation between miR-367 and Smad7 expression levels was determined by calculating the Spearman correlation coefficient. Overall survival was defined as the interval between the dates of surgery and death. The KaplanMeier method was used to compare overall survival among patients in different groups, and the log-rank test was used to estimate differences in survival. A $P$ value less than 0.05 was considered to be statistically significant.

\section{RESULTS}

Identification of miR-367 as a negative regulator of Smad7 in human pancreatic cancer. Three miRNA target prediction programmes, miRanda, TargetScan, and PicTar, were used to identify miRNA(s) that may be involved in the regulation of Smad7. Only miRNA target pairs, which bind to the same region in the target $3^{\prime}$-UTR sequence based on all three programmes, were selected in follow-up experiments (Xu et al, 2011). We found that 12 miRNAs including miR-96, $-182,-20 \mathrm{a},-106 \mathrm{a}, 367$, $-32,-25,-195,-15,-16,-21$, and $-181 \mathrm{a}$ had a potential binding site in the $3^{\prime}$-UTR of Smad7 (Supplementary Table 2). To investigate the roles of these predicted miRNAs in Smad7 expression, the miRNA mimics were co-transfected with a luciferase reporter construct containing wild-type Smad7 3'-UTR. As shown in Figure 1A, transfection with miR-106a, - 367, - 21, and -181 a mimics downregulated luciferase activity compared with transfection with a NC, suggesting that these four miRNAs are potential regulators of Smad7. Previous studies have shown that miR-106, -21, and -181a regulated the expression of Smad7 (Lin et al, 2014; Parikh et al, 2014; Smith et al, 2014; Wang et al, 2014a). However, the association between Smad7 and miR-367, which caused significant downregulation of luciferase activity, remains unknown. Therefore, we investigated the role of miR-367 in Smad7 expression in the present study. We co-transfected miR367 mimics or inhibitors with a luciferase reporter construct containing the wild-type Smad7 3 -UTR into human pancreatic cancer cell line PANC1. We found that miR-367 mimics inhibited luciferase activity in PANC1 cells in a dose-dependent manner. Conversely, the miR-367 inhibitor induced luciferase activity (Figure 1B). Next, we used a lentiviral infection system to establish PANC-1 and BxPC3 transfectants that stably expressed miR-367 (Supplementary Figure 1A,B). We found that miR-367 overexpression significantly suppressed expression of $\mathrm{Smad} 7$ at both mRNA and protein levels (Figure 1C). In addition, inhibition of endogenous miR-367 using a synthetic miR-367 inhibitor significantly increased Smad7 expression (Figure $1 \mathrm{D}$ and Supplementary Figure 1C). We then performed qRT-PCR assay to determine the expression levels of miR-367 and Smad7 in 10 human pancreatic cancer specimens. As expected, miR-367 expression was negatively associated with Smad7 mRNA level $(r=-0.674, \mathrm{P}=0.033$; Figure $1 \mathrm{E})$. We also evaluated the effects of the three other miRNAs including miR-21, -106a, and -181a on Smad7 expression in pancreatic cancer cells. We found that miR21 and miR-106a mimics also inhibited luciferase activity and suppressed the expression of Smad7 at mRNA and protein levels in PANC1 cells (Supplementary Figure 2A, B). In addition, the levels of miR-21 and miR-106a negatively correlated with Smad7 mRNA level in PDAC patients (Supplementary Figure 3). However, no significant regulatory effects of miR-181a on Smad7 expression were found in tested pancreatic cancer cells (Supplementary Figures 2 and 3).

miR-367 downregulated Smad7 expression by directly targeting its $3^{\prime}$-UTR. Next, we determined whether Smad7 was a direct 
A

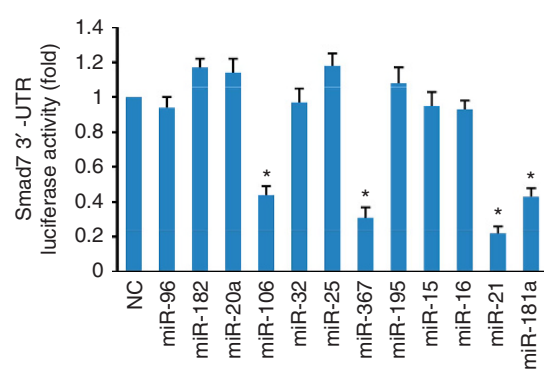

C

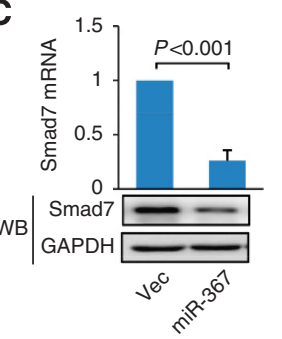

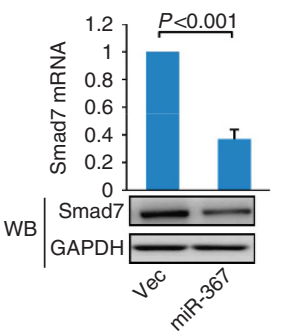

B

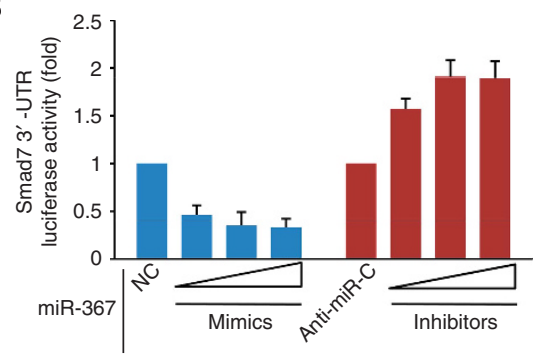

D

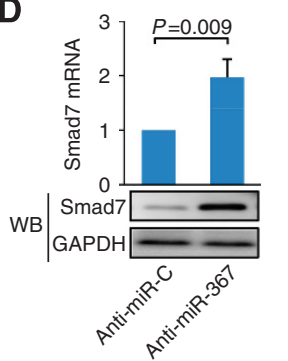

E

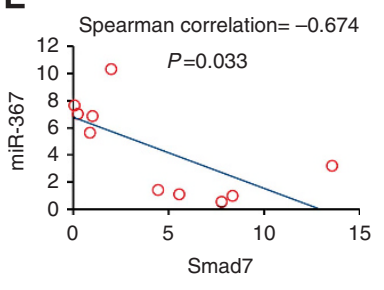

Figure 1. Identification of miR-367 as a negative regulator of Smad7 in human pancreatic cancer cells. (A) HEK293 cells were co-transfected with the negative control (NC) or miRNA mimics and the luciferase reporter construct containing the wild-type Smad7 3'-UTR. The results were normalised to the luciferase activity detected in hek293 cells transfected with the NC, and the luciferase activity of the NC was set to 1 . NS, not significant. Statistical significance was evaluated using ANOVA test. ${ }^{*} P<0.05$. (B) Mimics $(20,40$, and $60 \mathrm{nM})$ or inhibitors (60, 80, and 100 nM) of miR-367 were co-transfected into PANC-1 cells with the luciferase reporter construct containing the wild-type Smad7 3'-UTR. A scrambled mimic $(60 \mathrm{nM}, \mathrm{NC})$ or inhibitor (100 nM, Anti-miR-C) was used as a control. (C) Pancreatic cancer cells were infected with control or miR-367-expressing lentiviral vector. The expression levels of Smad7 mRNA and protein were evaluated using qRT-PCR and western blot analysis, respectively. (D) Pancreatic cancer cells were transiently transfected with anti-miR-367 inhibitor or anti-miR-C (NC). Twenty-four hours later, the expression levels of Smad7 mRNA and protein were evaluated using qRT-PCR and western blot analysis, respectively. Student's t-test was used to analyse the statistical significance of differences between groups. (E) The correlation between miR-367 and Smad7 mRNA expressions in pancreatic cancer biopsies obtained from pancreatic cancer patients $(n=10)$ was evaluated using Spearman's correlation analysis.

target of miR-367. Using the three miRNA target prediction programmes, we found that the $3^{\prime}$-UTRs of Smad7 mRNAs contain conserved miR-367-binding sites, which is highly conserved among humans, chimpanzees, mice, rats, and dogs (Figure 2A). A dual-luciferase reporter system was used to investigate whether miR-367 directly regulates Smad7 in pancreatic cancer (Figure 2B). We found that miR-367 expression significantly suppressed the firefly luciferase reporter activity of the wildtype Smad7 3'-UTR luciferase construct but not the mutant Smad7 $3^{\prime}$-UTR luciferase construct. These results were obtained from both HEK293 cells and the pancreatic cancer cell line PANC-1 (Figure 2C), suggesting that miR-367 suppresses Smad7 expression through directly targeting the miRNA-binding site in the Smad7 $3^{\prime}$-UTR.

miR-367 expression correlated with the prognosis of PDAC. Given that miR-367 negatively regulated Smad7, and low expression of Smad7 correlated with poor prognosis in PDAC patients (Wang et al, 2009b), we next investigated whether miR367 expression correlated with the prognosis of PDAC. The expression of miR-367 in 65 paraffin-embedded samples of PDAC tissues was evaluated using TaqMan quantitative real-time PCR. Kaplan-Meier analysis demonstrated that the median survival time of PDAC patients with low and high expression of miR-367 was 32.6 and 15.1 months, respectively (log-rank $=8.955, P=0.003$, Figure $3 \mathrm{~A}$ ). In addition, miR-367 expression in the cancer tissues with negative Smad7 expression was significantly higher than those with positive Smad7 expression, suggesting a negative correlation between miR-367 and Smad7 expressions (Figure 3B).

miR-367 promoted the invasion and metastasis of pancreatic cancer cells. We next evaluated the potential role of miR-367 in the growth of pancreatic cancer cells. We found that miR-367 overexpression had no significant effects on pancreatic cancer cell proliferation in vitro (Supplementary Figure 4A, B). However, miR-367 promoted the migration and invasion of PANC-1 and BxPC3 cells (Figure 4A, B). Furthermore, loss-of-function studies showed that silencing miR-367 with an inhibitor suppressed the migration and invasion of cancer cells (Figure 4C). To further investigate the effects of miR-367 overexpression on PDAC metastasis in vivo, we compared the metastatic nodules formed in the livers of NOD/SCID mice that were injected with tumour cells infected with either a miR-367-expressing lentiviral vector or an empty lentiviral vector. We found that both the control and miR-367-infected cells caused tumours after implanted into the spleen. miR-367 has little effects on the growth of spleen-implanted tumours (Supplementary Figure 4C), although tumours cells infected with miR-367-expressing lentiviral vector downregulated Smad7 expression (Supplementary Figure 4D). However, the mice injected with miR-367-overexpressing cells had increased numbers of liver metastatic colonies and an increased ratio of metastatic area to total area (Figure 4D) compared with mice injected with control cells. We additionally evaluated the effects of the other three miRNAs on cell invasion and found that miR-21 and -106a overexpression promoted PANC1 invasion, whereas miR-181a had no such effects (Supplementary Figure 5A, B). Taken together, these findings suggest that miR-367 promoted pancreatic cancer cell invasion and metastasis.

Smad7 mediated the invasion-promotion function of miR-367 in pancreatic cancer cells. To better understand the potential role of Smad7 in miR-367-mediated tumour invasion and metastasis, we explored the biological functions of Smad7 in human pancreatic cancer cells by transfecting PANC-1 and BxPC3 cells with a Smad7 siRNA. Our results showed that knockdown of endogenous Smad7 by siRNA significantly promoted the migration and invasiveness of PDAC cells (Figure 5A). This phenotype was similar to the effects 
A

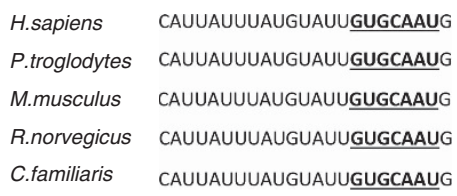

C

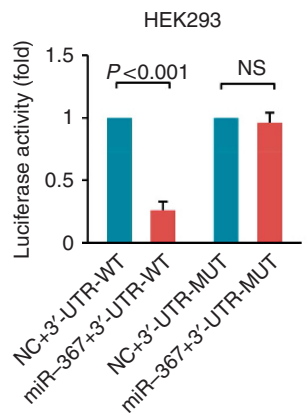

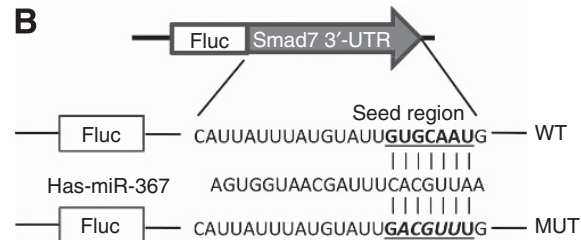

PANC-1

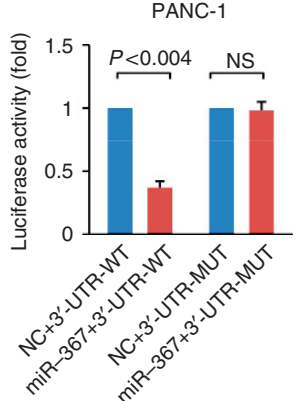

Figure 2. miR-367 downregulated Smad7 expression by directly targeting its $3^{\prime}$-UTR. (A) The sequences on Smad7 3'-UTR for potential binding with miR-367 were aligned among human (H. sapiens), chimpanzee ( $P$. troglodytes), mouse (M. musculus), rat (R. norvegicus), and dog (C. familiaris). Seed sequences were highlighted and underlined. (B) A human Smad7 3'-UTR fragment containing the wild-type or mutant miR-367-binding sequence was cloned downstream to the luciferase reporter gene. (C) HEK293 and PANC-1 cells were co-transfected with the negative control (NC) or miR-367 mimics and the luciferase reporter construct containing the wild-type or mutant Smad7 3'-UTR. For each experiment, the results were normalised to the luciferase activity detected in the cells transfected with the control vector. The luciferase activity of the control vector was set to 1 . NS, not significant. Statistical significance was evaluated using Student's t-test.

A

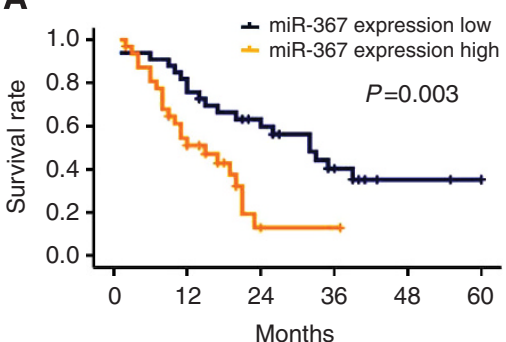

B

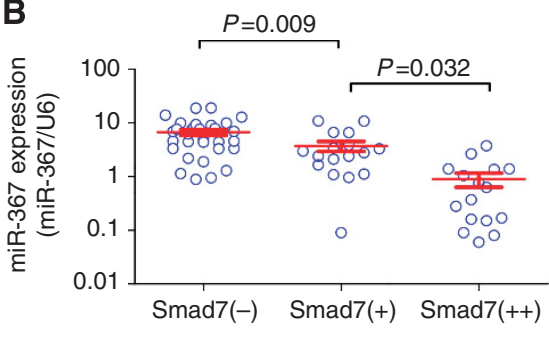

Figure 3. miR-367 expression correlated with the prognosis of PDAC. (A) Kaplan-Meier survival analysis of PDAC patients grouped by the expression levels of miR-367. The expression levels of mature miR-367 were evaluated by TaqMan real-time qPCR, and the median value of all 65 cases was defined as the cutoff value classified 65 tumour cases into low expression $(n=33)$ and high expression of miR-367 ( $n=32)$. (B) The correlation between the expressions of Smad7 and miR-367 was assessed in 65 PDAC patients. Smad7 protein expression was evaluated by immunohistochemistry (Wang et al, 2009b). Statistical analysis of the difference in miR-367 levels between Smad7( - ) and Smad7( + ) samples was conducted using ANOVA test.

induced by miR-367 overexpression. Then, we found that reintroduction of Smad7 without $3^{\prime}$-UTR into cancer cells led to constitutive expression of Smad7 without the binding site for miR367. We found that transfection with Smad7 without $3^{\prime}$-UTR significantly abrogated the increased migration and invasion of PANC1 cells induced by miR-367 (Figure 5B). Finally, cotransfection with anti-miR-367 and siSmad7 also showed that miR-367 silence could not repress the invasion in Smad7-depleted pancreatic cancer cells (Figure 5C). Therefore, these results suggest that Smad7 mediated the invasion-promotion function of miR-367 in pancreatic cancer cells, and Smad7 was a functional target of miR-367.

miR-367 promoted epithelial-to-mesenchymal transition (EMT) by increasing TGF- $\beta$-induced transcriptional activity. The TGF$\beta$ signalling pathway is involved in cell invasiveness and metastasis by inducing EMT (Wang et al, 2009a). Smad7 is a common inhibitory Smad that inhibits signal transduction triggered by TGF- $\beta$ s, activins, and BMPs (Hata et al, 1998; Ebisawa et al, 2001; Bai and Cao, 2002; Shi et al, 2004). Therefore, we investigated the effects of miR-367 on TGF- $\beta$ signalling activity and EMT in PDAC cells. We found that
miR-367 overexpression did not significantly alter the expressions of Smad2, Smad3, and Smad4 in PANC1 cells. However, miR-367 overexpression increased phosphorylated Smad2/3 upon TGF- $\beta 1$ stimulation (Figure 6A). To determine whether increased Smad2/3 phosphorylation induced by miR-367 overexpression increased the promoter activity of TGF- $\beta$, PANC1 cells were transfected with TGF- $\beta$ responsive p3TP-luciferase construct. Transfection with miR-367 in PANC1 cells significantly increased the luciferase activity, whereas transfection with Smad7 without $3^{\prime}$-UTR significantly abrogated the TGF- $\beta$ signalling activity induced by miR-367 (Figure 6B). EMT, in response to TGF- $\beta 1$, is phenotypically characterised by downregulation of a number of epithelial markers and upregulation of several mesenchymal markers (Pardali and Moustakas, 2007). In the present study, we found that miR-367 overexpression loosed cell contact, resulted in spindle-shaped morphology, reduced the expression of E-cadherin (an epithelial marker), and increased the expression of vimentin, a-SMA, and desmin (mesenchymal markers). However, transfection of Smad7 without $3^{\prime}$-UTR significantly abrogated the EMT induced by miR-367 (Figure 6C). Therefore, these results suggest that miR-367 promoted EMT by increasing TGF- $\beta$-induced transcriptional activity. 

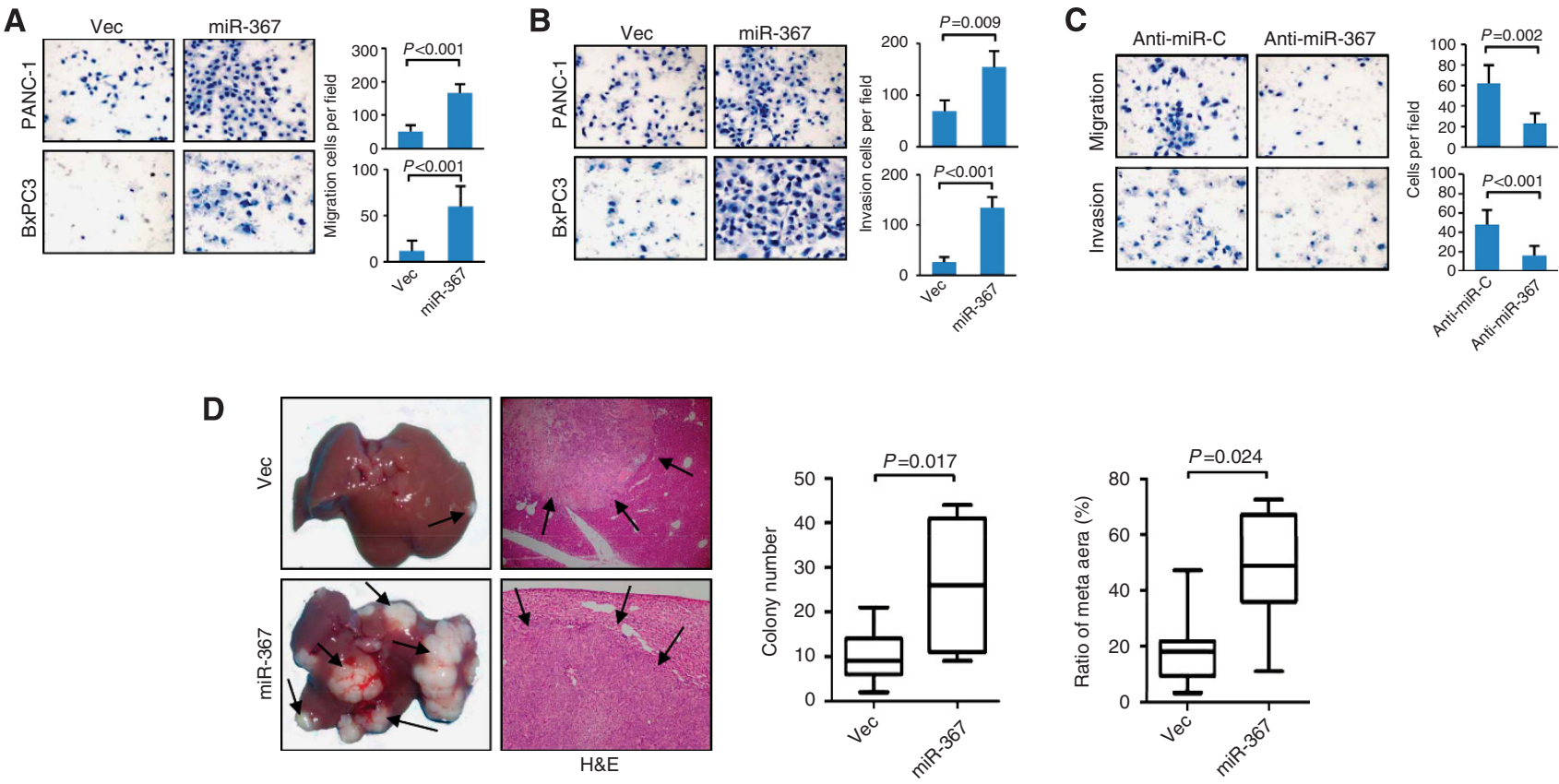

Figure 4. miR-367 promoted the invasion and metastasis of pancreatic cancer cells. (A) Migration and (B) invasion assays were performed using control lentiviral vector-infected or miR-367 vector-infected PANC-1 and BxPC3 cells. The number of cells that passed through the membrane was counted in 10 fields under the $\times 20$ objective lens, original magnification $(\times 200)$. The results obtained from three independent experiments were presented as the mean \pm s.d. of values. (C) Migration and invasion assays were performed using negative control (anti-miR-C) or anti-miR-367 inhibitor-transfected PANC-1 cells. The results obtained from three independent experiments were presented as the mean \pm s.d. of values. (D) Impact of miR-367 on tumour metastasis in vivo. Eight weeks after spleen injection of control vector-infected or miR-367 vector-infected cells, the mice were killed, and the livers were dissected. Representative livers from NOD/SCID mice were shown. H\&E staining was performed on sections of metastatic tumours and normal liver tissues. Arrows represent the metastatic nodules, original magnification ( $\times 200)$. Tumour metastases were quantified by counting the number of metastatic colonies in one histological section of the midportion of liver specimen from mice. The ratio of the metastatic area to the total area was determined in histological sections from the midportion of each liver (7 per group). Student's t-test was used to analyse the statistical significance of the differences between groups.
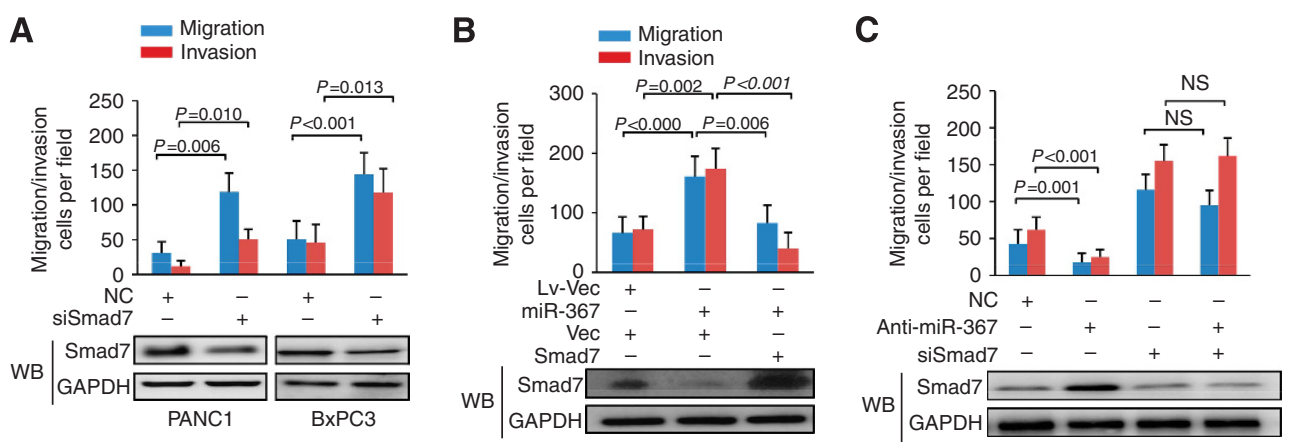

Figure 5. Smad7 mediated the invasion-promotion function of miR-367 in pancreatic cancer cells. (A) Migration and invasion assays of pancreatic cancer cells transfected with Smad7 siRNA. The expression level of Smad7 protein $24 \mathrm{~h}$ after transfection was evaluated by western blot analysis. The number of cells that passed through the membrane was counted in 10 fields under the $\times 20$ objective lens, original magnification ( $\times 200$ ). The results obtained from three independent experiments were presented as the mean \pm s.d. of values. (B) Migration and invasion assays of control vector-expressing and miR-367-expressing PANC1 cells transfected with a control vector or a Smad7-expressing vector that contained the entire coding sequence of Smad7 but lacked the 3'-UTR. The expression level of Smad7 protein was evaluated by western blot analysis. The number of cells that passed through the membrane was counted in 10 fields under the $\times 20$ objective lens, original magnification ( $\times 200$ ). The results obtained from three independent experiments were presented as the mean \pm s.d. of values. (C) Migration and invasion assays of PANC1 cells co-transfected with anti-miR-367 and siSmad7. Smad7 protein levels were evaluated by western blot analysis. The number of cells that invaded through the membrane was counted in 10 fields under the $\times 20$ objective lens., original magnification, $\times 200$. The results were presented as the mean \pm s.d. of values obtained from three independent experiments.

\section{DISCUSSION}

In our previous study, we found that Smad7 expression was associated with metastasis and prognosis of pancreatic duct adenocarcinoma. We showed that low level expression of Smad7 correlated with lymph node metastasis, liver metastasis after surgery, and a poor survival rate in pancreatic cancer patients, suggesting that aberrant Smad7 expression contributes to invasion and metastasis of pancreatic cancer cells. Here, we identified a miRNA, miR-367, and showed that miR-367 was involved in invasion and metastasis of pancreatic cancer cells by regulating Smad7 expression 
A

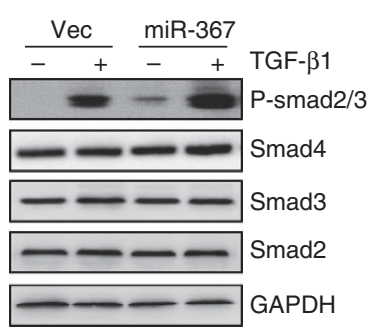

C

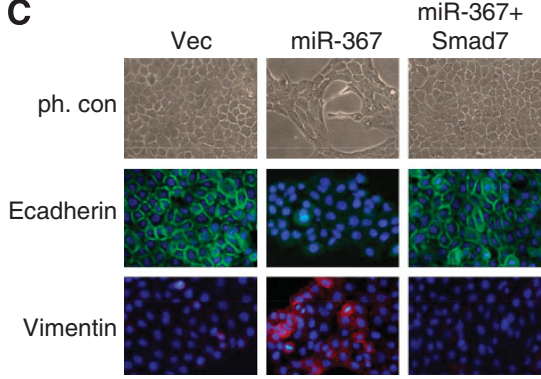

B
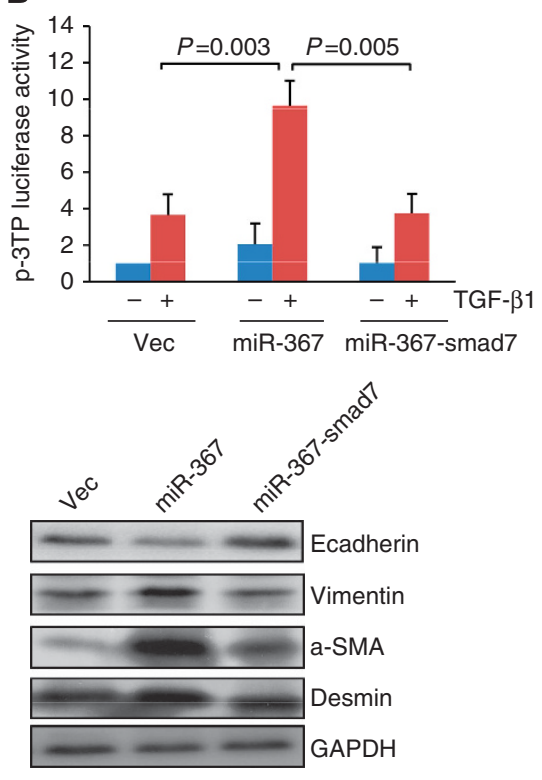

Figure 6. miR-367 promoted EMT by increasing TGF- $\beta$-induced transcriptional activity. (A) Control lentiviral vector-infected or miR-367 vectorinfected PANC-1 cells were uninduced or induced by TGF- $\beta 1(200 \mathrm{pM})$ for $1 \mathrm{~h}$. Whole-cell extracts were analysed by western blot analysis using antibodies against Smad2, Smad3, Smad4, and phosphorylated Smad2/3. GAPDH was used as a loading control. (B) Control vector-expressing and miR-367-expressing cells were co-transfected with a Smad7-expressing vector that contained the entire coding sequence of Smad7 but lacked the $3^{\prime}$-UTR and a TGF- $\beta$ responsive luciferase reporter, p3TP. TGF- $\beta$-induced luciferase activity was measured. The results obtained from three independent experiments were presented as the mean \pm s.d. of values. (C) BxPC3 cells were stained to detect E-cadherin and vimentin (green). Cell nuclei were counterstained with DAPI, original magnification $(\times 200)$. The morphology of cells was shown by phase-contrast images (ph.con.). Total protein was extracted, and the expressions of E-cadherin, vimentin, a-SMA, and desmin were evaluated by western blot analysis. GAPDH was used as a loading control.

Smad7 is a general inhibitory Smad that inhibits signal transduction triggered by TGF- $\beta$ (Hata et al, 1998; Ebisawa et al, 2001; Bai and Cao, 2002; Shi et al, 2004). Given that TGF- $\beta$ signalling has two opposite roles in tumour progression and metastasis, depending on the stage of carcinogenesis and the responsiveness of tumour cells (Akhurst and Derynck, 2001; Wakefield and Roberts, 2002), Smad7 may act as a tumourproliferation-promoting factor or a metastasis suppressor in human cancers. Previous studies have shown that the degree of Smad7 expression correlated with poor survival rates and more aggressive and invasive tumour behaviours in malignant follicular thyroid carcinomas, colorectal carcinomas, gastric carcinomas, and esophagus carcinomas (Boulay et al, 2003; Cerutti et al, 2003; Kim et al, 2004; Osawa et al, 2004). In addition, it has also been reported that Smad7 inhibited invasion and metastasis of xenografted breast cancer in mice (Azuma et al, 2005). Stable overexpression of Smad7 in human melanoma cells inhibited the metastasis of cancer in bone tissues (Javelaud et al, 2007). Therefore, the effect of Smad7 on cancer cell behaviours is dependent on the cancer cell responses to the TGF- $\beta /$ Smads signalling. In introductory studies, they have shown that in few pancreatic cancer cell lines growth was inhibited by TGF- $\beta$. In addition, the growth of most pancreatic cancer cell lines, including PANC-1, BxPC3, CF-Pac1, PT45, Hs766T, were not inhibited by TGF- $\beta$ (Kleeff and Korc, 1998; Nicolas and Hill, 2003; Subramanian et al, 2004). Although pancreatic cancer cells are not highly sensitive to TGF- $\beta$-induced growth inhibition, TGF- $\beta$ signalling may not be completely abrogated in pancreatic cancer cells. The TGF- $\beta$ signalling pathway is still functional, and TGF- $\beta$ serves as a tumour progression factor (Pardali and Moustakas, 2007). Therefore, in our previous study, we evaluated the effects of Smad7 in human pancreatic cancer and found that low expression of Smad7 correlated with lymph node metastasis, liver metastasis, and shortened survival time, suggesting that Smad7 is an invasion- and metastasis-suppressing factor.
In the present study, functional studies further confirmed that Smad7 inhibited migration and invasion of pancreatic cancer cells, and Smad7 may serve as a therapeutic target to prevent pancreatic cancer invasion and metastasis.

MiRNAs regulate the expression of target genes at posttranscriptional level. They degrade mRNAs and/or inhibit their translation by binding to partially complementary sequences in mRNAs, thereby downregulating protein expression (Kim et al, 2009). Recent study suggests that dysregulation of miRNAs is involved in many types of human diseases, including cancers (Iorio and Croce, 2009). For example, the function of mediating metastasis of pancreatic cancer has been assigned to dysregulated miRNAs (Papaconstantinou et al, 2012; Wang et al, 2014b). Our previous study has also demonstrated that dysregulated Smad7 was associated with tumour metastasis and poor prognosis of pancreatic cancer (Wang et al, 2009b). Whether Smad7 is posttranscriptionally regulated by miRNAs remains unclear. Therefore, in the present study, we aimed to identify miRNA(s) involved in the regulation of Smad7. We predicted miRNAs targeting Smad7 using miRNA prediction programmes followed by a luciferase reporter assay and identified four miRNAs that could regulate Smad7 among which, miR-106, -21, and -181 have been reported to directly regulate Smad7 by targeting its $3^{\prime}$-UTR. For miR-21 and miR-181c, both have been reported to enhance TGF$\beta 1$-induced EMT by targeting Smad7 in human cancers. Therefore, we focused on miR-367 that showed significant suppressing effects on luciferase activity of Smad7 $3^{\prime}$-UTR, which had never been reported for regulating Smad7 or affecting pancreatic cancer cell invasion. Our results confirmed ${ }^{* *}$ that miR-367 downregulated Smad7 expression in pancreatic cancer cells by directly targeting its 3'-UTR. In addition, as Smad7 has been shown to inhibit invasion of pancreatic cancer cells, we then showed that miR-367 promotes invasion and metastasis of human pancreatic cancer cells through regulating on the expression of Smad7. Therefore, we identifies an 
important miRNA, miR-367, that targets Smad7 and, thereby, promote pancreatic cancer invasion and metastasis.

Smad7 may negatively regulate the transcription of target gene induced by TGF- $\beta$. Previous studies have reported that the TGF- $\beta$ signalling pathway contributed to cell invasion and metastasis by inducing EMT, an important process observed in the invasion and metastasis of pancreatic cancer cells (Nakajima et al, 2004; Jungert et al, 2007). In the present study, we also evaluated the effects of miR-367 on TGFb/Smads signalling and EMT. We confirmed that miR-367 induced EMT by increasing TGF- $\beta$-induced transcriptional activity. Taken together, miR-367 induced EMT and promoted invasion and metastasis of pancreatic cancer cells through the regulation of Smad7 expression and the TGF- $\beta$ pathway.

In conclusion, we identified and characterised the miR-367Smad7-TGFb pathway, which is involved in invasion and metastasis of pancreatic cancer cells. Based on our results, miR367 may act as a therapeutic target for the treatment of human pancreatic cancer, especially cancers with high potential of invasion and metastasis.

\section{ACKNOWLEDGEMENTS}

This study was supported by National Science Foundation of China (81370068) and Natural Science Foundation of Shanghai (11ZR1407300).

\section{CONFLICT OF INTEREST}

The authors declare no conflict of interest.

\section{REFERENCES}

Akhurst RJ, Derynck R (2001) TGF-beta signaling in cancer-a double-edged sword. Trends Cell Biol 11: S44-S51.

Azuma H, Ehata S, Miyazaki H, Watabe T, Maruyama O, Imamura T, Sakamoto T, Kiyama S, Kiyama Y, Ubai T, Inamoto T, Takahara S, Itoh Y, Otsuki Y, Katsuoka Y, Miyazono K, Horie S (2005) Effect of Smad7 expression on metastasis of mouse mammary carcinoma JygMC(A) cells. J Natl Cancer Inst 97: 1734-1746.

Bai S, Cao X (2002) A nuclear antagonistic mechanism of inhibitory Smads in transforming growth factor-beta signaling. J Biol Chem 277: 4176-4182.

Boulay JL, Mild G, Lowy A, Reuter J, Lagrange M, Terracciano L, Laffer U, Herrmann R, Rochlitz C (2003) SMAD7 is a prognostic marker in patients with colorectal cancer. Int J Cancer 104: 446-449.

Cerutti JM, Ebina KN, Matsuo SE, Martins L, Maciel RM, Kimura ET (2003) Expression of Smad4 and Smad7 in human thyroid follicular carcinoma cell lines. J Endocrinol Invest 26: 516-521.

Ebisawa T, Fukuchi M, Murakami G, Chiba T, Tanaka K, Imamura T, Miyazono K (2001) Smurf1 interacts with transforming growth factor-beta type I receptor through Smad7 and induces receptor degradation. J Biol Chem 276: $12477-12480$.

Giovannetti E, Funel N, Peters GJ, Del Chiaro M, Erozenci LA, Vasile E, Leon LG, Pollina LE, Groen A, Falcone A, Danesi R, Campani D, Verheul HM, Boggi U (2010) MicroRNA-21 in pancreatic cancer: correlation with clinical outcome and pharmacologic aspects underlying its role in the modulation of gemcitabine activity. Cancer Res 70: 4528-4538.

Hata A, Lagna G, Massague J, Hemmati-Brivanlou A (1998) Smad6 inhibits BMP/Smad1 signaling by specifically competing with the Smad4 tumor suppressor. Genes Dev 12: 186-197.

Iorio MV, Croce CM (2009) MicroRNAs in cancer: small molecules with a huge impact. J Clin Oncol 27: 5848-5856.

Javelaud D, Delmas V, Moller M, Sextius P, Andre J, Menashi S, Larue L, Mauviel A (2005) Stable overexpression of Smad7 in human melanoma cells inhibits their tumorigenicity in vitro and in vivo. Oncogene 24: 7624-7629.
Javelaud D, Mohammad KS, McKenna CR, Fournier P, Luciani F, Niewolna M, Andre J, Delmas V, Larue L, Guise TA, Mauviel A (2007) Stable overexpression of Smad7 in human melanoma cells impairs bone metastasis. Cancer Res 67: 2317-2324.

Jungert K, Buck A, von Wichert G, Adler G, Konig A, Buchholz M, Gress TM, Ellenrieder V (2007) Sp1 is required for transforming growth factor-betainduced mesenchymal transition and migration in pancreatic cancer cells. Cancer Res 67: 1563-1570.

Kim VN, Han J, Siomi MC (2009) Biogenesis of small RNAs in animals. Nat Rev Mol Cell Biol 10: 126-139.

Kim YH, Lee HS, Lee HJ, Hur K, Kim WH, Bang YJ, Kim SJ, Lee KU, Choe KJ, Yang HK (2004) Prognostic significance of the expression of Smad4 and Smad7 in human gastric carcinomas. Ann Oncol 15: $574-580$.

Kleeff J, Korc M (1998) Up-regulation of transforming growth factor (TGF)-beta receptors by TGF-beta1 in COLO-357 cells. J Biol Chem 273: 7495-7500.

Lekanne Deprez RH, Fijnvandraat AC, Ruijter JM, Moorman AF (2002) Sensitivity and accuracy of quantitative real-time polymerase chain reaction using SYBR green I depends on CDNA synthesis conditions. Anal Biochem 307: 63-69.

Lim LP, Lau NC, Garrett-Engele P, Grimson A, Schelter JM, Castle J, Bartel DP, Linsley PS, Johnson JM (2005) Microarray analysis shows that some microRNAs downregulate large numbers of target mRNAs. Nature 433: 769-773.

Lin L, Gan H, Zhang H, Tang W, Sun Y, Tang X, Kong D, Zhou J, Wang Y, Zhu Y (2014) MicroRNA21 inhibits SMAD7 expression through a target sequence in the 3' untranslated region and inhibits proliferation of renal tubular epithelial cells. Mol Med Rep 10: 707-712.

Nakajima S, Doi R, Toyoda E, Tsuji S, Wada M, Koizumi M, Tulachan SS, Ito D, Kami K, Mori T, Kawaguchi Y, Fujimoto K, Hosotani R, Imamura M (2004) N-cadherin expression and epithelial-mesenchymal transition in pancreatic carcinoma. Clin Cancer Res 10: 4125-4133.

Nicolas FJ, Hill CS (2003) Attenuation of the TGF-beta-Smad signaling pathway in pancreatic tumor cells confers resistance to TGF-beta-induced growth arrest. Oncogene 22: 3698-3711.

Osawa H, Nakajima M, Kato H, Fukuchi M, Kuwano H (2004) Prognostic value of the expression of Smad6 and Smad7, as inhibitory Smads of the TGF-beta superfamily, in esophageal squamous cell carcinoma. Anticancer Res 24: 3703-3709.

Papaconstantinou IG, Lykoudis PM, Gazouli M, Manta A, Polymeneas G, Voros D (2012) A review on the role of microRNA in biology, diagnosis, and treatment of pancreatic adenocarcinoma. Pancreas 41: 671-677.

Pardali K, Moustakas A (2007) Actions of TGF-beta as tumor suppressor and pro-metastatic factor in human cancer. Biochim Biophys Acta 1775: 21-62.

Parikh A, Lee C, Peronne J, Marchini S, Baccarini A, Kolev V, Romualdi C, Fruscio R, Shah H, Wang F, Mullokandov G, Fishman D, D'Incalci M, Rahaman J, Kalir T, Redline RW, Brown BD, Narla G, DiFeo A (2014) microRNA-181a has a critical role in ovarian cancer progression through the regulation of the epithelial-mesenchymal transition. Nat Commun 5: 2977.

Shi W, Sun C, He B, Xiong W, Shi X, Yao D, Cao X (2004) GADD34-PP1c recruited by Smad7 dephosphorylates TGFbeta type I receptor. J Cell Biol 164: 291-300.

Siegel R, Ma J, Zou Z, Jemal A (2014) Cancer statistics, 2014. CA Cancer J Clin 64: 9-29.

Smith AL, Iwanaga R, Drasin DJ, Micalizzi DS, Vartuli RL, Tan AC, Ford HL (2014) The miR-106b-25 cluster targets Smad7, activates TGF-beta signaling, and induces EMT and tumor initiating cell characteristics downstream of Six1 in human breast cancer. Oncogene 31: 5162-5171.

Subramanian G, Schwarz RE, Higgins L, McEnroe G, Chakravarty S, Dugar S, Reiss M (2004) Targeting endogenous transforming growth factor beta receptor signaling in SMAD4-deficient human pancreatic carcinoma cells inhibits their invasive phenotype1. Cancer Res 64: 5200-5211.

Wakefield LM, Roberts AB (2002) TGF-beta signaling: positive and negative effects on tumorigenesis. Curr Opin Genet Dev 12: 22-29.

Wang JY, Gao YB, Zhang N, Zou DW, Wang P, Zhu ZY, Li JY, Zhou SN, Wang SC, Wang YY, Yang JK (2014a) miR-21 overexpression enhances TGF-beta1-induced epithelial-to-mesenchymal transition by target smad7 and aggravates renal damage in diabetic nephropathy. Mol Cell Endocrinol 392: 163-172.

Wang P, Chen L, Zhang J, Chen H, Fan J, Wang K, Luo J, Chen Z, Meng Z, Liu L (2014b) Methylation-mediated silencing of the miR-124 genes 
facilitates pancreatic cancer progression and metastasis by targeting Rac1. Oncogene 33: 514-524.

Wang P, Chen Z, Meng ZQ, Fan J, Luo JM, Liang W, Lin JH, Zhou ZH, Chen H, Wang K, Shen YH, Xu ZD, Liu LM (2009a) Dual role of Ski in pancreatic cancer cells: tumor-promoting versus metastasis-suppressive function. Carcinogenesis 30: 1497-1506.

Wang P, Fan J, Chen Z, Meng ZQ, Luo JM, Lin JH, Zhou ZH,

Chen H, Wang K, Xu ZD, Liu LM (2009b) Low-level expression of Smad7 correlates with lymph node metastasis and poor prognosis in patients with pancreatic cancer. Ann Surg Oncol 16: 826-835.
Xu Z, Xiao SB, Xu P, Xie Q, Cao L, Wang D, Luo R, Zhong Y, Chen HC, Fang LR (2011) miR-365, a novel negative regulator of interleukin-6 gene expression, is cooperatively regulated by Sp1 and NF-kappaB.J Biol Chem 286: 21401-21412.

This work is published under the standard license to publish agreement. After 12 months the work will become freely available and the license terms will switch to a Creative Commons AttributionNonCommercial-Share Alike 4.0 Unported License.

Supplementary Information accompanies this paper on British Journal of Cancer website (http://www.nature.com/bjc) 